\title{
Clarifying some issues on the geoeffectiveness of limb halo CMEs
}

\author{
Consuelo Cid ${ }^{1}$, Hebe Cremades ${ }^{2}$, Angels Aran ${ }^{3}$, Cristina Mandrini ${ }^{4,5}$, \\ Blai Sanahuja ${ }^{3}$, Brigitte Schmieder ${ }^{6}$, Michel Menvielle ${ }^{7,8}$, Luciano \\ Rodriguez $^{9}$, Elena Saiz ${ }^{1}$, Yolanda Cerrato ${ }^{1}$, Sergio Dasso ${ }^{10,4}$, \\ Carla Jacobs ${ }^{11}$, Chantal Lathuillere ${ }^{12}$, and Andrei Zhukov ${ }^{9}$ \\ ${ }^{1}$ Departamento de Física y Matemáticas, Universidad de Alcalá, \\ Campus Universitario, A- II, km. 33,600, E-28871 Alcalá de Henares (Madrid), Spain \\ email: consuelo.cid@uah.es \\ ${ }^{2}$ Universidad Tecnológica Nacional-Facultad Regional Mendoza /CONICET, \\ Rodriguez 243, M5502AJE, Mendoza, Argentina \\ ${ }^{3}$ Departament d' Astronomia i Meteorologia and Institut de Ciències del Cosmos, Universitat \\ de Barcelona, Martí i Franquès 1, E-08028 Barcelona, Spain \\ ${ }^{4}$ Instituto de Astronomía y Física del Espacio, IAFE, CONICET-UBA, \\ CC 67 Suc. 28, 1428 Buenos Aires, Argentina \\ ${ }^{5}$ Facultad de Ciencias Exactas y Naturales, UBA, Buenos Aires, Argentina \\ ${ }^{6}$ Observatoire de Paris, LESIA, 92290, Meudon, France \\ ${ }^{7}$ CNRS/INSU, Universitè Versailles St-Quentin, \\ LATMOS-IPSL, Guyancourt, France \\ ${ }^{8}$ Univ Paris-Sud, Dèpartement des Sciences de la Terre, Orsay, France \\ ${ }^{9}$ Solar-Terrestrial Center of Excellence SIDC, \\ Royal Observatory of Belgium, Av. Circulaire 3, 1180, Brussels, Belgium \\ ${ }^{10}$ Departamento de Física, Facultad de Ciencias Exactas y Naturales, \\ UBA, Buenos Aires, Argentina \\ ${ }^{11}$ Space Applications Services NV \\ Leuvensesteenweg 325, B-1932 Zaventem, Belgium \\ ${ }^{12}$ UJF-Grenoble 1 / CNRS-INSU, Institut de Planètologie et d'Astrophysique de Grenoble \\ (IPAG) UMR 5274, Grenoble, F-38041, France
}

\begin{abstract}
A recent study by Cid et al. (2012) showed that full halo coronal mass ejections (CMEs) coming from the limb can disturb the terrestrial environment. Although this result seems to rise some controversies with the well established theories, the fact is that the study encourages the scientific community to perform careful multidisciplinary analysis along the Sunto-Earth chain to fully understand which are the solar triggers of terrestrial disturbances. This paper aims to clarify some of the polemical issues arisen by that paper.
\end{abstract}

Keywords. Sun: coronal mass ejections (CMEs), Sun: solar-terrestrial relations

\section{Introduction}

Since the beginning of the $80 \mathrm{~s}$, when Howard et al. (1982) reported the observation of a coronal transient directed at Earth as a bright halo around the coronagraphs of LASCO instrument on board SoHO, the term 'halo CME' has been associated with an ejection of material from the solar corona with a large probability to disturb the terrestrial environment, especially if it travels fast and if the magnetic field embebed on the interplanetary counterpart is properly oriented. However, both terms ('CME' and 
'halo') are only related to the way something is observed, i.e., the term 'CME' is not related to material from the solar corona, but to the ejection recorded by an instrument designed to observe the solar corona, i.e., a coronagraph (Hudson et al. 2006). Also the term 'halo' does not mean material directed towards or away from the Earth, but just a bright halo that appears to surround the occulting disk of a coronagraph.

A very simple model for a full halo CME is that proposed by Howard et al. (1982), where the observed bright halo will consist on the 2D projection of solar material propagating approximately towards or away from the Earth, i.e., from the observer. In this model, a solar source close to the solar central meridian appears as a condition to the full halo signature. Otherwise, the solar source should be located at the back side and the CME will travel away from our planet. In this scenario, no full halo CME should arise close to the limb, and all full halo CMEs with a solar source close to the solar central meridian should reach the Earth. However, it is easy to find examples to prove that both statements are far to be right and that full halo CMEs close to the limb should not be discarded as potential hazards to the terrestrial environment.

An additional issue is to estimate the time of arrival of a solar ejection at the Earth. There are several CME and interplanetary shock propagation models, both physic-based and empirical (see for example Gopalswamy et al. 2001, Fry et al. 2003 and references therein). Checking the prediction capability of some of these models, Cho et al. (2003) obtained that there were $20 \%$ of cases where the predictions exceeded 24 hours in errors. Among others, they proposed as possible reasons for this large uncertainty a complex heliospheric environment involving interactions between shocks or interplanetary CMEs, or even some misidentifications, i.e., a wrong linkage between the solar source and the interplanetary consequence. In fact these two reasons might have also been involved in the different results obtained by analyzing the geoeffectiveness of halo CMEs on the basis of a large set of solar observations from statistical analysis. Just as an example, for a similar period of time, Gopalswamy et al. (2007) found that geoeffectiveness of frontside halo CMEs was $71 \%$ while Kim et al. (2005) obtained $40 \%$.

\section{Full halo CMEs with a solar source close to the solar limb}

Figure 1 shows C2 and EIT images during four full halo CMEs that disturbed the Earth during solar cycle 23 (Cid et al. 2012). The solar sources of these events are listed in Table 1. In all cases their position is less than 10 degrees far from the solar limb. Three of them originate from the West limb and one is from the East limb, showing a western bias already found by Wang et al. (2002). These events not only prove the existence of full halo CMEs from the limb but all of them also present two common signatures:

(1) they are associated with flares of X (or almost) class (see Table 1) and

(2) they are highly asymmetric and they exhibit a bright feature close to the limb where the solar source is located.

The first signature, where three X-class X-ray flares and one M9.1 are related to the CMEs on Figure 1, indicates a huge amount of energy released. This suggests that the active region where both the flare and the CME originate is able to produce powerful events. It is so, that the large brightening coming from the limb is able to surround the whole coronagraph resulting in a full halo CME. This kind of events are far from the 2D projection model proposed by Howard et al. (1982).

The second signature indicates a large difference between these limb halo CMEs and the 'gradually expanding, Sun-centered excess brightness' originated by the sudden disappearance of a large filament at N05W03 and a relatively minor solar flare at N18E05, reported by Howard et al. (1982). This asymmetric excess brightness relative to solar 
Table 1. Geoeffective LFH CMEs of solar cycle 23.

\begin{tabular}{|c|c|c|c|c|c|c|c|c|c|}
\hline CME date & DoY & $\mid \underset{[\mathrm{UT}]}{\mathbf{C M E} \text { time }}$ & $\mid \begin{array}{c}\text { CME speed } \\
{[\mathrm{km} / \mathrm{s}]}\end{array}$ & $\left|\begin{array}{c}\text { F lare } \\
\text { class }\end{array}\right|$ & $\begin{array}{c}\text { Flare max } \\
{[\mathrm{UT}]}\end{array}$ & $\begin{array}{l}\text { Solar } \\
\text { source }\end{array}$ & $\left|\begin{array}{c}\text { Flare } \\
\text { location }\end{array}\right|$ & $\mid \begin{array}{c}\mathbf{D s t}_{\text {min }} \\
{[\mathrm{nT}]}\end{array}$ & $\mid \begin{array}{l}\text { Dst }_{\text {min }} \\
{[\mathrm{DoY} / \mathrm{hh}]}\end{array}$ \\
\hline 01102001 & 274 & | 05:30:05 & 1405 & | M9.1 | & $05: 15$ & AR 9628 & S18W90 | & 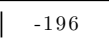 & 27615 \\
\hline | 21042002 & | 111 | & | $01: 27: 20$ & 2393 & $|\mathrm{X} 1.5|$ & $01: 51$ & A R 9906 & S14W 84 & -57 & 11316 \\
\hline 15062003 & 166 & | 23:54:05 & 2053 & $|\mathrm{X} 1.3|$ & $23: 56$ & | AR 10386 & S07E80 | & | -141 & | 16910 \\
\hline | 04112003 & 308 & | 19:54:05 & 2657 & $\mathrm{X} 28$ & $19: 50$ & | AR 10486 & S19W 83 & -33 & 31320 \\
\hline
\end{tabular}

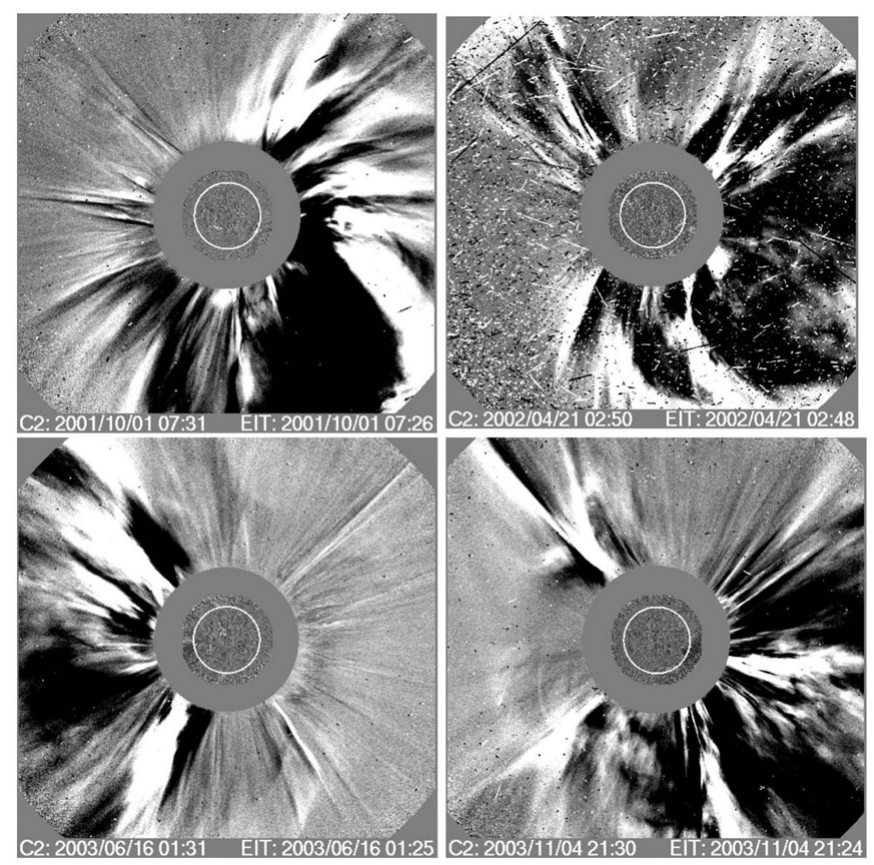

Figure 1. Four limb full halo CMEs that disturbed the terrestrial environment.

center, shown in the coronagraph images in Figure 1, may be used as an indicator of the location of the solar source. Moreover, the direction of maximum brightness might be related to the propagation direction, therefore, the direction of the interplanetary counterpart of the CME can be guessed (Cremades and Bothmer 2005).

\section{Reaching the terrestrial environment from the solar limb}

A controversial issue still remains in the scenario described above, regarding the fact that the interplanetary counterpart of a CME from the limb could reach the Earth and disturb it. The key point to solve the problem is to consider that disturbing the terrestrial environment does not mean that the ejected solar material during the CME reaches the Earth. But, how is it possible to disturb the Earth without reaching it? As indicated in Table 1, the speed of the four limb full halo CMEs of solar cycle 23 which are geoeffective is really large. As a result, all of them drive fast interplanetary shocks. Using Helios spacecraft data, de Lucas et al. (2011) found that 50\% of CME driven shocks can be found generally to manifest themselves over ecliptic longitudinal distances of $90^{\circ}$. Considering the expansion in longitude of shock waves, de Lucas et al. (2011) concluded that one can expect with about a $50 \%$ chance that the shock driven by a limb CME will 
hit the Earth. Indeed, not only the shock driven by the CME but even the turbulent extended sheath behind the shock may disturb the terrestrial environment.This seems to be what happened in the case of the four full halo CMEs from Table 1, although only $16 \%$ of the limb halo CMEs analyzed by Cid et al. (2012) disturbed the Earth.

Moreover, Cid et al. (2012) showed that to assess the severity of a geomagnetic storm, not only the Dst index needs to be checked but also other geomagnetic indices have to be analysed because the $D s t$ is only a proxy for the disturbances occurring at low latitudes. As an example, after the CME on November 4, 2003 the Dst and $S Y M-H$ indices did not fall below the $-50 \mathrm{nT}$ threshold. However, this CME is related to an active period (starting with a sharp increase in $S Y M-H$ at around 19:40 UT on November 6) preceded and followed by periods of low magnetic activity in other indices as $A S Y-H$, $A L$ or $a m$, which clearly indicate a disturbed terrestrial environment.

As a final issue, we insist on the difficulty in tracking any event from its solar source up to its terrestrial consequences. The footprints at different stages are usually difficult to connect. Frequently, interplanetary Type II emissions and solar energetic particle data provide a way to glue some pieces but sometimes to choose among the different possibilities to fix the puzzle requires a thorough study to come up with a solution. Broadly speaking, the correct choice to build up the whole Sun-to-Earth chain may be quite different from that obtained by merely applying the time-window method commonly used in statistical studies. Detailed studies are the unique procedure to reach the right linkage among the solar source, the interplanetary counterpart and the terrestrial consequences, and even in this case evidences collected may not be enough to uniquely solve the problem.

\section{Acknowledgements}

The authors would like to thank to the International Space Science Institute (Bern, Switzerland) for supporting the project "From the Sun to the terrestrial surface: understanding the chain", led by C. Cid and all data sources used in this work. C.C. thanks IAU for the support to participate in the IAUS300 Symposium.

\section{References}

Cho, K.-S. et al. 2003, J. Geophys.Res., 108, 1445, doi:10.1029/2003JA010029

Cid, C., et al. 2012, J. Geophys. Res., 117, A11102, doi:10.1029/2012JA017536

Cremades, H. \& Bothmer, V. 2005, Coronal and Stellar Mass Ejections Proceedings IAU Symposium, No. 226 , 2005, K. P. Dere, J. Wang \& Y. Yan, eds., doi:10.1017/S174392130500013X

de Lucas, A., et al. 2011, J. Atm. Sol. Terr. Phys., 73, 1281, doi:10.1016/j.jastp.2010.12.011

Fry, C. D., et al. 2003, J. Geophys. Res., 108 , 1070, doi:10.1029/2002JA009474

Gopalswamy, N., Lara, A., Yashiro, S., Kaiser, M., \& Howard R. A. 2001, J. Geophys. Res., 106, 29,207, doi:10.1029/2001JA000177

Gopalswamy, N., Yashiro, S., \& Akiyama, S. 2007, J. Geophys. Res., 112, A06112, doi:10.1029/2006JA012149

Howard, R. A., Michels, D. J., Sheeley Jr., N. R., \& Koomen, M. J. 1982, Ap. J. Let., 263, 10.1

Hudson, H. S., Bougeret, J.-L., \& Burkepile, J. 2006, Space Sc. Rev., 123, 13

Kim, R.-S. et al. 2005, J. Geophys. Res., 110, A11104, doi:10.1029/2005JA011218

Wang, Y. M., Ye, P. Z., Wang, S., Zhou, G. P., \& Wang, J. X. 2002, J. Geophys. Res., 107, 1340, doi:10.1029/2002JA009244 